\title{
Fatores associados ao óbito de mulheres com notificação de violência por parceiro íntimo no Brasil
}

\author{
Factors associated with death in women with intimate partner \\ violence notification in Brazil
}

Isabella Vitral Pinto (https://orcid.org/0000-0002-3535-7208) 1,2

Regina Tomie Ivata Bernal (https://orcid.org/0000-0002-7917-3857) ${ }^{3}$

Maria de Fátima Marinho de Souza (https://orcid.org/0000-0003-3287-9163) ${ }^{4}$

Deborah Carvalho Malta (https://orcid.org/0000-0002-8214-5734) ${ }^{5}$
${ }^{1}$ Programa de PósGraduação em Saúde Pública, Universidade Federal de Minas Gerais (UFMG). Av. Prof. Alfredo Balena 190, Centro. 30130-100 Belo Horizonte MG Brasil. isabella.pinto@fiocruz.br ${ }^{2}$ Instituto René Rachou, Fiocruz Minas. Belo Horizonte MG Brasil. ${ }^{3}$ Faculdade de Saúde Pública, Universidade de São Paulo. São Paulo SP Brasil.

${ }^{4}$ Vital Strategies Brasil. São

Paulo SP Brasil.

${ }^{5}$ Departamento de Enfermagem Materno Infantil e Saúde Pública, Escola de Enfermagem, UFMG. Belo Horizonte MG Brasil.

\begin{abstract}
Intimate partner violence (IPV) is a social and public health concern. This article aims to identify factors associated with death in women with IPV notification. This is a case-control study based on the record-linkage of the Mortality Information System (SIM), from 2011 to September/2017, and the Notifiable Diseases Information System (SINAN), 2011 to 2016. The "case" group consisted of women aged 15 to 59 years old with notification of violence on SINAN, whose author was current or former intimate partner and died by any cause registered on SIM. The "control" group consisted of women 15-59 years old, living in the same municipality of the cases and victim of IPV registered on SINAN, but without a death record on SIM. Multiple logistic regression was used to estimate the odds ratio of death. 151,826 Brazilian women were victim of IPV and were notified by SINAN, and 2,538 died. The main cause of death was homicide. The following characteristics conferred a greater chance of death: having race/skin-color black or yellow; having disabilities; living in rural area; physical violence, torture and multiple types combined; violence perpetrated by a firearm, a sharp object and multiple means combined.

Key words Intimate partner violence, Gender-based violence, Public health surveillance, Epidemiological monitoring
\end{abstract}

Resumo A violência por parceiro intimo (VPI) é um problema social e de saúde pública. O objetivo deste artigo é identificar fatores associados ao óbito em mulheres com notificação de VPI. Estudo do tipo caso-controle a partir do relacionamento das bases de dados do Sistema de Informação sobre Mortalidade (SIM), 2011 a setembro/2017, e do Sistema de Informação de Agravos de Notificação (SINAN), 2011 a 2016. O grupo "caso" foi composto por mulheres de 15 a 59 anos com notificação de violência no SINAN, cujo autor era atual ou ex-parceiro intimo e óbito por qualquer causa básica registrado no SIM. O grupo "controle" consistiu em mulheres da mesma faixa etária, com VPI registrada no SINAN e município de residência dos casos, mas sem registro de óbito no SIM. Foi utilizada a regressão logística a fim de estimar a razão de chance de óbito. 151.826 brasileiras tiveram seus casos de VPI notificados, sendo que 2.538 morreram. A principal causa de óbito foi o homicídio. As seguintes características conferiram maior chance de óbito: raça/cor preta e amarela; ter deficiências; residir em área rural; violência fisica, tortura e múltiplos tipos combinados; violenncia perpetrada por arma de fogo, objeto perfurocortante e múltiplos meios combinados.

Palavras-chave Violência por parceiro intimo, Violência de gênero, Vigilância em saúde pública, Monitoramento epidemiológico 


\section{Introdução}

Rosa, Maria, Angélica, elas, nós. A violência por parceiro íntimo (VPI) é uma experiência que atravessa a história das mulheres, seja porque já a vivenciamos ou porque temos alguma situação na família, entre amigas ou conhecidas. A partir das lutas dos movimentos feministas em defesa dos direitos das mulheres, o assunto tem ganhado espaço nas leis, agendas políticas, noticiários e no nosso dia a dia.

Esse problema tem escala global e a importância do seu enfrentamento foi concretizado com indicadores específicos na Agenda 2030', onde os 193 Estados Membros da Organização das Nações Unidas se comprometeram a alcançar as metas relacionadas à equidade de gênero. Estudo de revisão sistemática com artigos do período de 1990 a 2011, de diversos países e continentes, demonstrou que $38,6 \%$ de todos os homicídios contra mulheres foram perpetrados por parceiros íntimos ${ }^{2}$. Considerando que esses feminicídios não são um fato isolado na vida das mulheres, mas representam o ponto final em uma sequência de violências e abusos, pode-se inferir que entre os óbitos e as vivências da VPI, há um grande iceberg a ser descortinado. No Brasil, estudo com amostra representativa da cidade de São Paulo e de 15 municípios da Zona da Mata de Pernambuco identificou prevalência, pelo menos uma vez na vida, de mais de $41 \%$ de violência psicológica e de mais de $27 \%$ de violência física por parceiro íntimo entre mulheres ${ }^{3}$.

A VPI é também um problema de saúde pública, pois os abusos físicos, psicológicos, sexuais e negligências decorrentes dessas agressões aparecem como demandas principais e em queixas difusas nos atendimentos do setor. Além disso, a saúde é um componente fundamental para a integralidade do cuidado, seja por meio da recuperação do estado físico e psicológico, ou pelos encaminhamentos que se fazem necessários a outros órgãos responsáveis pela garantia dos direitos das mulheres.

Com intuito de ampliar as possibilidades de diagnóstico e monitoramento desses eventos, o Ministério da Saúde criou em 2006, o Sistema Nacional de Vigilância de Violência e Acidentes (VIVA), cujo componente contínuo prevê a notificação compulsória de violências interpessoais e autoprovocadas nos serviços públicos e privados de todo o país a partir de $2011^{4,5}$. Essa notificação tem o intuito de dar visibilidade ao problema, atuar como um instrumento de cuidado e promover atuação integrada entre os órgãos da rede de atendimento às mulheres em situação de violência.

Portanto, faz-se necessário compreender: O que acontece às mulheres após a notificação? Elas permanecem vivas ou morrem? Quais são as principais causas básicas de óbito? Quais são os fatores associados ao óbito?

A declaração de óbito é o instrumento de coleta padronizada dos dados sobre mortalidade e não contém informação sobre o sexo do autor da agressão e tipo de vínculo com a vítima. No entanto, é possível recontar parte da história das mulheres a partir do cruzamento dos registros de notificação de violência e do óbito.

Diante desse quadro, propõe-se explorar os fatores associados ao óbito em mulheres com notificação de violência por parceiro íntimo. Com isso pretende-se identificaras variáveis que possam sinalizar cenários de maior risco e gerar alerta para prevenir óbitos prematuros e evitáveis.

\section{Métodos}

Estudo do tipo caso-controle realizado a partir do relacionamento das bases de dados do Sistema de Informação sobre Mortalidade (SIM), com 3.492.636 registros de óbitos de pessoas do sexo feminino, de todas as idades, no período de 2011 a setembro/2017; e do Sistema de Informação de Agravos de Notificação (SINAN), com 812.157 notificações de violências interpessoais e autoprovocadas em pessoas do sexo feminino, de todas as idades, no período de 2011 a 2016.

O relacionamento das bases do SIM e SINAN foi conduzido pelo Ministério da Saúde ${ }^{6}$, sendo utilizada a técnica dos Filtros de Bloom e as seguintes variáveis de pareamento: "nome da paciente", "data de nascimento" e "município de residência". Os pares com pontuação igual a 10.000 (valor máximo) foram considerados verdadeiros e aqueles com pontuação entre 9.000 e 9.999 passaram por avaliação manual, na qual também foi utilizada a variável "nome da mãe" para análise ${ }^{6}$. Como resultado, foram identificados $19.604 \mathrm{pa}$ res considerados como verdadeiros, os quais representaram 17.566 mulheres com notificação de violência que foram a óbito no período avaliado (Figura 1).

Também foi necessário avaliar na base do SINAN a presença de mulheres com uma ou mais notificações no período. Para isso, foi realizada uma análise, também conduzida pelo Ministério da Saúde, utilizando a técnica dos Filtros de Bloom. Nesta nova etapa os registros do SINAN 


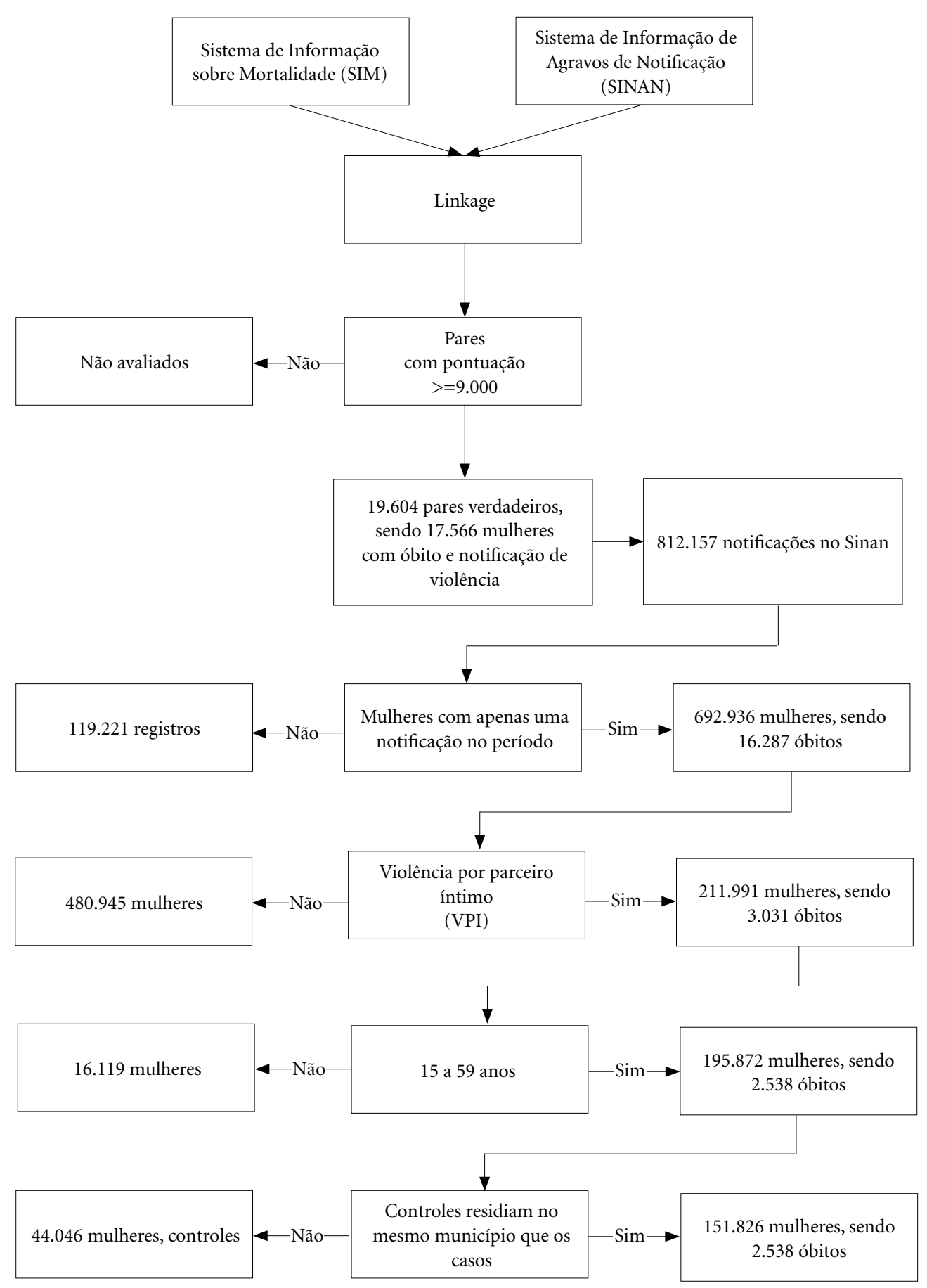

Figura 1. Fluxograma do relacionamento das bases de dados do Sistema de Informação sobre Mortalidade (SIM), no período de 2011 a setembro/2017, e do Sistema de Informação de Agravos de Notificação (SINAN), no de 2011 a 2016, Brasil.

Fonte: Sistema de Informação sobre Mortalidade (SIM) e Sistema de Informação de Agravos de Notificação (SINAN), Ministério da Saúde. 
foram agrupados a partir das variáveis "nome da paciente", "data de nascimento", "município de residência" e "nome da mãe", buscando identificar pares ou grupos de mulheres similares. Os grupos com pontuação menor que 9.000 representaram 692.936 registros $(85,3 \%$ das notificações) e foram considerados únicos, ou seja, representaram mulheres com uma notificação no período. Os outros 119.221 registros com pontuação maior ou igual a 9.000 foram relacionados em pares ou grupos que poderiam representar a mesma mulher. No entanto, esses registros não foram avaliados (Figura 1).

Portanto, este estudo considerou como ponto de partida as 692.936 mulheres com uma notificação de violência no período avaliado. Destacase que as variáveis analisadas foram provenientes do SINAN, exceto a causa básica do óbito, originária do SIM. Selecionaram-se os registros com os seguintes vínculos/grau de parentesco entre o agressor e a pessoa atendida: "cônjuge", "ex-cônjuge", "namorado" e "ex-namorado"; também foram incluídos os casos com vínculos similares a essas quatro categorias no campo aberto "outros" dessa variável. Foram excluídas as notificações com violência autoprovocada ou com informações sobre tentativa de suicídio ou autoagressão no campo aberto "Tipo de Violência - Outros". Por fim, para promover maior similaridade entre casos e controles, foram selecionadas somente as mulheres jovens e adultas com idade de 15 a 59 anos, e aquelas residentes nos 1.104 municípios em que os casos residiam quando foi registrada a notificação (Figura 1).

O grupo dos casos foi composto por mulheres de 15 a 59 anos com notificação de violência no SINAN, cujo autor era atual ou ex-parceiro íntimo, e que veio a óbito por qualquer causa básica registrada no SIM. O grupo controle consistiu em mulheres com a mesma faixa etária, notificação de VPI no SINAN e mesmo município de residência dos casos, mas sem registro de óbito no SIM no período avaliado (Figura 1).

Descreveu-se as causas básicas de óbito segundo a Classificação Internacional de Doenças -10a Edição (CID-10), organizadas em grupos de causas, os quais estão disponíveis para acesso no tabulador eletrônico do Departamento de Informática do SUS ${ }^{7,8}$.

$\mathrm{Na}$ ficha de notificação de violências no SINAN, nas variáveis "Tipo de Violência" e "Meio de Agressão" é possível assinalar mais de uma opção, resultando em diversas possibilidades de combinações. Portanto, foi necessário propor nova organização das variáveis, a fim de garantir que cada mulher fosse identificada com uma única categoria. Como critério para a criação dessas novas variáveis foram consideradas a frequência das categorias e hierarquias de importância com base nas repercussões na saúde, conforme apresentado abaixo.

As combinações encontradas de tipos de violência foram dispostas em: psicológica (somente esse tipo); física (somente esse tipo); psicológica e física (somente esses dois tipos); sexual (somente esse tipo ou todas as combinações em que ele esteja presente); tortura (somente esse tipo ou todas as combinações em que ele esteja presente, exceto as combinações da categoria anterior); financeira (somente esse tipo ou todas as combinações em que ele esteja presente, exceto as combinações das duas categorias anteriores); outras (somente esse tipo ou todas as outras categorias possíveis ou seus cruzamentos, exceto as combinações das três categorias anteriores); ignorado (quando não foi assinalada qualquer categoria de tipo de violência).

Para a variável "meios de agressão" as novas categorias resultaram em: "ameaça" (somente esse meio); "força física" (somente esse meio); "ameaça e força física" (somente esses dois meios); "arma de fogo" (somente esse meio e todas as combinações em que ele esteja presente); "objeto perfurocortante" (somente esse meio ou todas as combinações em que ele esteja presente, exceto as combinações da categoria anterior); "enforcamento" (somente esse meio ou todas as combinações em que ele esteja presente, exceto as combinações das duas categorias anteriores); "objeto contundente" (somente esse meio ou todas as combinações em que ele esteja presente, exceto as combinações das três categorias anteriores); "outros" (somente esse meio ou todas as outras categorias possíveis ou seus cruzamentos, exceto as combinações das quatro categorias anteriores); ignorado (quando não foi assinalada qualquer categoria de meio de agressão).

Procedeu-se a análise descritiva de 12 variáveis categóricas da ficha de notificação, segundo o desfecho (não óbito; e óbito): faixa etária; gestante; raça/cor; escolaridade; situação conjugal; deficiência; zona de residência; porte do município de residência, de acordo com o número de habitantes; local da ocorrência; violência crônica (se ocorreu mais de uma vez); tipo de violência; e meio de agressão. A categoria "missing" foi incluída na categoria "ignorado" de cada variável. Também se realizou o Teste Qui-Quadrado $\left(\chi^{2}\right)$ para comparar as proporções entre os dois grupos avaliados. 
Como neste universo o grupo de casos (2.538 mulheres) tem número proporcionalmente pequeno em relação ao grupo de controles (149.288 mulheres), utilizou-se a técnica de validação cruzada $^{9}$ mediante geração de amostras de treino e teste para construção do modelo, na proporção de dois controles para cada caso, a fim de encontrar o modelo robusto e com capacidade de generalização para evitar o super ajuste (overfitting) do modelo. Foram geradas de forma aleatória 10 amostras de treino e as respectivas 10 amostras de teste, representativas do universo.

Para cada amostra de treino foi realizada a análise de regressão logística, com as 12 variáveis independentes relacionadas à notificação de violência no SINAN, a fim de estimar a razão de chance de óbito. Utilizou-se o método stepwise para seleção das variáveis do modelo final. Foram avaliadas a multicolinearidade, por meio do teste de fator de inflação da variância (VIF), e o ajuste do modelo, por meio do teste de Hosmer e Lemenshow. O modelo final de cada amostra de treino foi aplicado à respectiva amostra de teste.

A amostra de treino que apresentou maior equilíbrio entre especificidade, sensibilidade e acurácia, na comparação com a amostra de teste, foi utilizada para estimar as razões de chance entre os grupos expostos e não expostos, segundo os desfechos de óbito e não óbito. Todas as análises foram conduzidas no software R Studio ${ }^{10}$.

Este estudo faz parte do projeto "Como morrem as mulheres com notificação de violência por parceiro íntimo no Brasil?”, aprovado pelo Comitê de Ética em Pesquisa da Universidade Federal de Minas Gerais.

\section{Resultados}

Entre as 692.936 mulheres com uma notificação de violência interpessoal ou autoprovocada, $16.287(2,4 \%)$ foram a óbito, por diversas causas, entre janeiro de 2011 a setembro de 2017. Esse fato representou a morte de mais de seis mulheres com uma notificação de violência a cada dia no Brasil, no período avaliado. A partir desse universo, foram selecionadas as notificações de VPI (211.991); as mulheres entre 15 e 59 anos (195.872); e os municípios de residência das mulheres que foram a óbito. Portanto, este estudo trabalhou com 151.826 mulheres, sendo 149.288 do grupo controle e 2.538 do grupo caso (Figura $1)$.

Os dez principais grupos de causas básicas de óbito das mulheres foram: "Agressões", tota- lizando 1.030 óbitos (40,6\%); “Neoplasias malignas" com 196 óbitos (7,7\%); "Doença pelo vírus da imunodeficiência humana (HIV)" com 125 óbitos (4,9\%); "Acidentes de transporte" com 110 óbitos (4,3\%); "Doenças cerebrovasculares" com 96 óbitos (3,8\%); "Causas mal definidas e desconhecidas de mortalidade" com 89 óbitos (3,5\%); "Doença isquêmica do coração" com 84 óbitos (3,3\%) ; "Doenças do fígado" com 78 óbitos (3,1\%); "Eventos (fatos) cuja intenção é indeterminada" com 76 óbitos (3,0\%); e "Outras causas externas de traumatismos acidentais" com 64 óbitos (2,5\%).

A comparação da distribuição das características relativas às mulheres e aos eventos na ficha de notificação mostram características particulares do grupo que foi a óbito no período máximo de 6,9 anos após a notificação: 25,97\% de jovens entre 15 a 29 anos; $46,70 \%$ eram mulheres negras, considerando pretas e pardas; e 8,67\% eram mulheres com deficiência (Tabela 1 ). No grupo com desfecho "óbito" foi maior o percentual de mulheres com menor escolaridade (Tabela 1).

A avaliação dos municípios e zonas de residência no momento da notificação evidenciou a maior vulnerabilidade das mulheres que moravam em áreas rurais e periurbanas e em municípios de pequeno porte $(<50.000$ habitantes), totalizando $11,58 \%$ e $36,96 \%$ dos óbitos, respectivamente (Tabela 1 ).

$\mathrm{Na}$ análise das variáveis relativas à violência notificada, destacam-se as seguintes características no grupo cujo desfecho foi o óbito: o principal local da ocorrência da violência foi a residência $(74,78 \%)$; a violência física preponderou em $57,21 \%$ dos casos; e os percentuais expressivos de agressões perpetradas com objetos perfurocortantes $(23,84 \%)$ e armas de fogo $(10,68 \%)$. A violência crônica foi observada em $45,43 \%$ das mulheres com óbito, no entanto, essa variável apresentou mais de $25 \%$ de casos ignorados ( Tabela 1).

A amostra de treino selecionada para representar o universo apresentou $72 \%$ de sensibilidade, $71 \%$ de especificidade e $72 \%$ de acurácia. Nessa amostra de treino, o modelo da regressão logística apresentou as seguintes variáveis significativas, ao nível de significância de 0,05: faixa etária (30 a 44; 45 a 59); gestante (sim); raça/cor (preta e amarela); escolaridade (ensino médio e ensino superior - completos ou incompletos); deficiência (sim); zona de residência (rural); porte do município de residência (todas as categorias); local de ocorrência da violência (residência e ignorado); violência crônica (ignorado); 
Tabela 1. Distribuição percentual das características das mulheres com notificação de violência por parceiro íntimo e das violências registradas, segundo óbito e não óbito, Brasil, 2011 a 2016.

\begin{tabular}{|c|c|c|c|c|c|c|c|}
\hline \multirow[t]{2}{*}{ Características } & Não óbito & Óbito & $\begin{array}{c}\text { Teste } \\
\mathbf{X}^{2}\end{array}$ & \multirow[t]{2}{*}{ Características } & \multirow{2}{*}{$\begin{array}{l}\text { Não óbito } \\
\mathrm{N}=149.288\end{array}$} & \multirow{2}{*}{$\begin{array}{c}\text { Óbito } \\
\mathrm{N}=2.538\end{array}$} & \multirow{2}{*}{$\begin{array}{c}\text { Teste } \\
\mathbf{X}^{2} \\
\text { p-valor }\end{array}$} \\
\hline & $\mathrm{N}=149.288$ & $\mathrm{~N}=2.538$ & p-valor & & & & \\
\hline Faixa etária (anos) & & & & Porte do município de & & & \\
\hline 15 a 29 & 12,44 & 25,97 & $<0,01$ & residência & & & \\
\hline 30 a 44 & 41,94 & 42,91 & & $<10.000$ & 1,60 & 9,26 & $<0,01$ \\
\hline 45 a 59 & 45,62 & 31,13 & & $\geq 10.00$ e $<50.000$ & 14,14 & 27,70 & \\
\hline Gestante & & & & $\geq 50.00$ e $<100.000$ & 13,93 & 14,34 & \\
\hline Não & 64,62 & 68,60 & $<0,01$ & $\geq 100.00$ e $<500.000$ & 38,57 & 29,47 & \\
\hline Sim & 6,50 & 4,49 & & $\geq 500.000$ & 31,76 & 19,23 & \\
\hline Ignorado & 28,88 & 26,91 & & Local da Ocorrência & & & \\
\hline Raça/Cor & & & & $\begin{array}{l}\text { Via pública, bar ou } \\
\text { comércio/serviços }\end{array}$ & 12,91 & 15,56 & 0,01 \\
\hline Branca & 43,46 & 41,57 & $<0,01$ & Residência & 77,89 & 74,78 & \\
\hline Preta & 9,72 & 11,82 & & Outros $^{2}$ & 3,45 & 4,49 & \\
\hline Amarela & 0,66 & 1,10 & & Ignorado & 5,76 & 5,16 & \\
\hline Parda & 33,73 & 34,87 & & Violência crônica & & & \\
\hline Indígena & 0,57 & 0,59 & & Não & 25,17 & 29,20 & $<0,01$ \\
\hline Ignorado & 11,86 & 10,05 & & Sim & 57,38 & 45,43 & \\
\hline Escolaridade & & & & Ignorado & 17,45 & 25,37 & \\
\hline Analfabeto & 0,83 & 2,88 & $<0,01$ & Tipos de violência & & & \\
\hline Ensino & 33,29 & 40,50 & & Psicológica & 12,40 & 4,89 & $<0,01$ \\
\hline Fundamental $^{1}$ & & & & & & & \\
\hline Ensino Médio $^{1}$ & 28,32 & 17,02 & & Física & 44,15 & 57,21 & \\
\hline Ensino Superior ${ }^{1}$ & 6,02 & 2,40 & & Física+psicológica & 31,52 & 21,75 & \\
\hline Ignorado & 31,53 & 37,19 & & Sexual $^{3}$ & 4,25 & 3,86 & \\
\hline Estado civil & & & & Tortura $^{3}$ & 3,63 & 7,09 & \\
\hline Solteira & 30,34 & 26,32 & $<0,01$ & Financeira $^{3}$ & 2,68 & 1,34 & \\
\hline Casada & 51,09 & 53,19 & & Outras $^{3}$ & 0,88 & 2,76 & \\
\hline Separada & 9,82 & 10,91 & & Ignorado & 0,48 & 1,10 & \\
\hline Viúva & 0,62 & 1,58 & & Meios de Agressão & & & \\
\hline Ignorado & 8,13 & 8,00 & & Ameaça & 11,27 & 4,85 & $<0,01$ \\
\hline Deficiência & & & & Força & 44,33 & 31,05 & \\
\hline Não & 82,74 & 75,37 & $<0,01$ & Força+ameaça & 13,83 & 7,88 & \\
\hline Sim & 3,13 & 8,67 & & Arma de fogo ${ }^{3}$ & 1,16 & 10,68 & \\
\hline Ignorado & 14,13 & 15,96 & & $\begin{array}{l}\text { Objeto pérfuro- } \\
\text { cortante }^{3}\end{array}$ & 7,66 & 23,84 & \\
\hline Zona de residência & & & & Enforcamento $^{3}$ & 6,40 & 5,20 & \\
\hline Urbana & 91,72 & 85,70 & $<0,01$ & Objeto contundente ${ }^{3}$ & 3,97 & 5,28 & \\
\hline $\begin{array}{l}\text { Rural ou } \\
\text { periurbana }\end{array}$ & 5,40 & 11,58 & & Outros meios ${ }^{3}$ & 6,52 & 7,09 & \\
\hline Ignorado & 2,89 & 2,72 & & Ignorado & 4,87 & 4,14 & \\
\hline
\end{tabular}

tipo de violência (física, sexual, tortura e outras) e meio de agressão (arma de fogo, objeto perfu- rocortante, objeto contundente e outros meios) (Tabela 2). 
Tabela 2. Modelo final de regressão logística múltipla, a partir da amostra de treino selecionada. Brasil, 2011 a 2016.

\begin{tabular}{|c|c|c|c|c|c|c|}
\hline Características & $\beta$ & $\begin{array}{c}\text { Erro } \\
\text { Padrão }\end{array}$ & p-valor & $\begin{array}{l}\text { Odds } \\
\text { Ratio }\end{array}$ & $\begin{array}{l}\text { Limite } \\
\text { inferior } \\
(\mathrm{IC} 95 \%)\end{array}$ & $\begin{array}{c}\text { Limite } \\
\text { superior } \\
\text { (IC95\%) }\end{array}$ \\
\hline \multicolumn{7}{|l|}{ Faixa etária (anos) } \\
\hline 15 a 29 & & & & 1,00 & & \\
\hline 30 a 44 & $-0,85$ & 0,09 & $<0,01$ & 0,43 & 0,25 & 0,61 \\
\hline 45 a 59 & $-1,28$ & 0,10 & $<0,01$ & 0,28 & 0,09 & 0,47 \\
\hline \multicolumn{7}{|l|}{ Gestante } \\
\hline Não & & & & 1,00 & & \\
\hline Sim & $-0,45$ & 0,16 & $<0,01$ & 0,64 & 0,33 & 0,95 \\
\hline \multicolumn{7}{|l|}{ Raça/Cor } \\
\hline Branca & & & & 1,00 & & \\
\hline Preta & 0,25 & 0,12 & 0,03 & 1,29 & 1,06 & 1,52 \\
\hline Amarela & 1,32 & 0,36 & $<0,01$ & 3,76 & 3,06 & 4,46 \\
\hline \multicolumn{7}{|l|}{ Escolaridade } \\
\hline Analfabeto & & & & 1,00 & & \\
\hline Ensino Médio $^{1}$ & $-0,82$ & 0,26 & $<0,01$ & 0,44 & $-0,08$ & 0,96 \\
\hline Ensino Superior ${ }^{1}$ & $-1,53$ & 0,32 & $<0,01$ & 0,22 & $-0,4$ & 0,84 \\
\hline \multicolumn{7}{|l|}{ Deficiência } \\
\hline Não & & & & 1,00 & & \\
\hline Sim & 0,78 & 0,14 & $<0,01$ & 2,19 & 1,91 & 2,47 \\
\hline \multicolumn{7}{|l|}{ Local da Ocorrência } \\
\hline Via pública, bar ou comércio/serviços & & & & 1,00 & & \\
\hline Residência & $-0,35$ & 0,10 & $<0,01$ & 0,71 & 0,51 & 0,9 \\
\hline Ignorado & $-0,58$ & 0,18 & $<0,01$ & 0,56 & 0,21 & 0,91 \\
\hline \multicolumn{7}{|l|}{ Violência crônica } \\
\hline Não & & & & 1,00 & & \\
\hline Ignorado & 0,36 & 0,11 & $<0,01$ & 1,43 & 1,22 & 1,64 \\
\hline \multicolumn{7}{|l|}{ Tipos de violência } \\
\hline Psicológica & & & & 1,00 & & \\
\hline Física & 0,90 & 0,19 & $<0,01$ & 2,45 & 2,09 & 2,82 \\
\hline Física+psicológica & 0,37 & 0,19 & 0,05 & 1,44 & 1,07 & 1,81 \\
\hline Sexual $^{2}$ & 0,77 & 0,23 & $<0,01$ & 2,16 & 1,71 & 2,61 \\
\hline Tortura $^{2}$ & 1,20 & 0,23 & $<0,01$ & 3,33 & 2,87 & 3,78 \\
\hline Outras $^{2}$ & 1,64 & 0,30 & $<0,01$ & 5,19 & 4,56 & 5,75 \\
\hline \multicolumn{7}{|l|}{ Meios de Agressão } \\
\hline Ameaça & & & & 1,00 & & \\
\hline Arma de fogo ${ }^{2}$ & 2,53 & 0,26 & $<0,01$ & 12,52 & 12,02 & 13,03 \\
\hline Objeto pérfuro-cortante ${ }^{2}$ & 1,14 & 0,20 & $<0,01$ & 3,14 & 2,74 & 3,53 \\
\hline Objeto contundente $^{2}$ & 0,54 & 0,23 & 0,02 & 1,71 & 1,25 & 2,16 \\
\hline Outros meios ${ }^{2}$ & 0,75 & 0,19 & $<0,01$ & 2,12 & 1,74 & 2,5 \\
\hline \multicolumn{7}{|l|}{ Zona de residência } \\
\hline Urbana & & & & 1,00 & & \\
\hline Rural ou periurbana & 0,35 & 0,13 & 0,01 & 1,42 & 1,17 & 1,67 \\
\hline \multicolumn{7}{|l|}{ Porte do município de residência } \\
\hline$<10.000$ & & & & 1,00 & & \\
\hline$\geq 10.00$ e $<50.000$ & $-1,06$ & 0,19 & $<0,01$ & 0,35 & $-0,02$ & 0,72 \\
\hline$\geq 50.00 \mathrm{e}<100.000$ & $-1,61$ & 0,20 & $<0,01$ & 0,20 & $-0,18$ & 0,58 \\
\hline$\geq 100.00$ e $<500.000$ & $-1,73$ & 0,19 & $<0,01$ & 0,18 & $-0,19$ & 0,54 \\
\hline$\geq 500.000$ & $-1,92$ & 0,19 & $<0,01$ & 0,15 & $-0,23$ & 0,52 \\
\hline
\end{tabular}


De acordo com esse modelo, observa-se que as mulheres em faixas etárias mais velhas (30 a 44; e 45 a 59 anos) têm chance menor de óbito em comparação com as jovens (15 a 29 anos). As mulheres grávidas no momento da notificação de violência tiveram menor chance de óbito em comparação às não grávidas. Esse resultado não esperado pode ter relação com a alta proporção de casos ignorados nesta variável. As mulheres pretas e amarelas tiveram chance 1,29 vezes e 3,76 vezes maior de óbito, respectivamente, em comparação às brancas. A presença de ensino médio ou superior, completos ou incompletos, representou uma proteção para as mulheres em situação de VPI. A presença da deficiência foi considerada um fator de risco para as mulheres, já que elas tiveram 2,19 vezes mais chance de óbito em comparação com aquelas sem deficiência. As mulheres que habitavam em áreas rurais ou periurbanas no momento da notificação da violência no SINAN tiveram chance 1,42 vezes mais elevada de óbito em comparação com aquelas residentes em áreas urbanas. Em consonância com o resultado anterior, quanto maior o porte do município de residência, maior a proteção das mulheres com relação ao óbito, em comparação com aquelas que moravam em municípios de até 10.000 habitantes. A ocorrência da violência na residência e em locais ignorados pelos profissionais que preencheram a ficha de notificação, aparecem como fatores protetores para o óbito em comparação às violências ocorridas em locais públicos como via pública, bar ou comércio/ serviços. Nos casos em que o caráter crônico da VPI foi ignorado, a chance de óbito das mulheres foi 1,43 vezes maior. Com relação aos tipos de violência, a presença da violência física "somente" e todas as combinações identificadas foram consideradas fator de risco para o óbito, em comparação com a psicológica "somente" $\mathrm{O}$ fator associado com maior risco de óbito para as mulheres foi a utilização de arma de fogo, com 12,52 vezes maior chance de óbito em relação às mulheres cuja violência foi perpetrada por meio de ameaças. Outros fatores de risco para o óbito foram os objetos perfurocortantes, contundentes e a combinação de outros meios (Tabela 2).

\section{Discussão}

Rosa, Maria e Angélica são mulheres do século XVIII, viviam no estado de São Paulo, e tiveram suas histórias reveladas por Mary del Priore ${ }^{11}$. As situações de ameaça, violência física, psicológica e negligência perpetradas por seus companheiros, contra elas e seus filhos, evidenciam como a violência baseada no gênero possui raízes profundas na constituição da nossa sociedade.

No presente há outros meios de revelar essas histórias: as mídias, os registros dos serviços da rede de atendimento, como os boletins de ocorrência e as notificações de violência na saúde, e as declarações de óbito. Os dados do setor saúde são essenciais para identificar as mulheres em situação de risco de feminicídio íntimo ${ }^{12}$. Além disso, há instrumentos legais, como a Lei Maria da Penha, para combater, punir e oferecer proteção às mulheres em situação de violência. No entanto, a ocorrência do feminicídio íntimo, crime cometido contra mulheres cujo agressor teve ou tem uma relação íntima, de afeto ou sexual ${ }^{13}$, é sinal de falha das instituições públicas, privadas e da sociedade nos campos da prevenção de violências, proteção às mulheres e na garantia de seus direitos.

Este artigo apresentou um retrato de mais de 151 mil brasileiras que tiveram seus casos de VPI registrados pelas unidades de saúde no SINAN. Apesar do poder público ter ciência da situação de vulnerabilidade a que estavam submetidas, 2.538 mulheres morreram no período de 6,9 anos após a notificação. A principal causa de óbito foi o homicídio, o que sugere possível ocorrência de feminicídio íntimo, especialmente pelo fato de a literatura apontar o parceiro íntimo como principal autor dos homicídios de mulheres ${ }^{2,14}$. Nesse campo de estudo, trabalha-se com esse tipo de estimativa pois a informação sobre o vínculo/grau de parentesco dos autores das agressões não está presente na declaração de óbito, sendo necessárias investigações para compreender as circunstâncias e contexto do crime ${ }^{13}$.

Algumas doenças crônicas não transmissíveis e a infecção pelo vírus HIV figuraram entre as causas mais prevalentes, denotando possíveis impactos da VPI no cuidado de condições crônicas. O estudo do Ministério da Saúde que utilizou essa mesma base de dados demonstrou uma maior razão de mortalidade para doenças crônicas não transmissíveis em mulheres com notificação de violência registrada no SINAN ${ }^{6}$. De fato, é reconhecido o efeito negativo das violências contra as mulheres nos cuidados à saúde que demandam acompanhamento contínuo, sendo inclusive fator de risco para a inadequação ao rastreio de câncer de colo do útero ${ }^{15}$. A literatura tem demonstrado também que, além das lesões físicas, a VPI tem sido relacionada ao maior risco de transmissão de infecções sexualmente transmissíveis, incluindo o HIV, e a efeitos na saúde mental, como perda da 
autoestima, ansiedade, depressão e síndrome do estresse pós traumático ${ }^{16}$.

De modo geral, no momento da notificação, as seguintes características estiveram associadas ao óbito: ser da raça/cor preta e amarela; ter deficiências; residir em área rural; residir em município de pequeno porte $(<10.000$ habitantes); estar em situação de violência física, tortura e múltiplos tipos combinados; a violência ter sido perpetrada por arma de fogo, objeto perfurocortante e múltiplos meios combinados.

A vivência de VPI muitas vezes começa na adolescência ou na juventude e perpassa diferentes classes sociais ${ }^{17}$. Na vivência do ficar e namorar as mulheres se deparam com os padrões de gênero nas relações afetivas e sexuais, o que pode gerar conflitos e agressões de diferentes tipos. Estudo sobre as notificações de violências em mulheres no período de 2011 a 2017 identificou que $41,3 \%$ das violências registradas em adolescentes de 15 a 19 anos foram perpetradas por parceiros íntimos ${ }^{18}$, mostrando uma frequência muito alta desse problema em uma população tão jovem, em construção da sua identidade e muitas vezes sem autonomia financeira.

No caso da raça/cor, o racismo estrutural e as iniquidades no acesso aos serviços de saúde e garantia de direitos podem estar relacionados à maior chance de as mulheres pretas terem o desfecho de óbito em relação às brancas. Os dados apresentados neste estudo mostraram que as mulheres grávidas no momento da notificação tiveram chances menores de óbito, ou seja, a gravidez apareceu como um fator de proteção para o óbito. No entanto, esse resultado pode ter sido impactado pelos problemas de completude dessa variável. Portanto, faz-se necessário estudo específico para avaliar a relação da VPI na gravidez e o desfecho de óbito. De toda forma, sabe-se que as violências na gestação causam repercussões no acesso das mulheres aos serviços de saúde, ao acompanhamento no pré-natal e puerpério, assim como tem consequências negativas na saúde do bebê $\hat{1}^{19,20}$.

A maior escolaridade foi fator de proteção para a ocorrência de óbito após a notificação de VPI. Esse fato revela a importância do acesso à educação para meninas e mulheres que passam a ter maiores condições de identificar as violências sofridas e buscar os serviços especializados que podem apoiá-las. Além disso, a maior escolaridade promove a autonomia financeira das mulheres e consequentemente, facilita o rompimento com os relacionamentos abusivos.

As mulheres com deficiências que tiveram notificação de VPI apresentaram maior chance de óbito, o que revela situações de vulnerabilidades que se somam. Faz-se necessário, futuramente, explorar as diferentes deficiências e suas associações com este desfecho.

A residência em zona rural apareceu como fator associado ao óbito. Possivelmente as mulheres que habitam em zonas rurais têm maior dificuldade de acesso aos serviços de saúde e da rede de serviços que atendem mulheres em situação de violência. Somado a isso, a distância de vizinhos, a menor densidade demográfica, além de padrões culturais e sociais patriarcais favorecem a invisibilidade do problema na comunidade.

Nesse mesmo sentido, residir em municípios com maior população foi associado à proteção com relação ao óbito. Pode-se inferir que em municípios com maior população espera-se contar com serviços de referência no campo da saúde, assistência social e segurança pública. Essa situação pode favorecer o acesso à rede de atendimento e da sociedade civil no sentido de buscar atendimento, proteção e até abrigamento nos casos de risco de morte.

Mais de 74\% das violências, nos dois grupos (óbito e não óbito) ocorreram na residência, desvelando como o ambiente doméstico pode representar risco para as mulheres. Apesar desse fato, as mulheres com notificação de VPI na residência parecem estar mais protegidas do óbito do que aquelas com notificação de VPI em locais públicos. Isso pode ser devido ao fato de a exposição pública das agressões revelar um estágio mais grave e crônico de violência.

Apesar do estudo trabalhar somente com mulheres com uma notificação no período avaliado, a variável da ficha de notificação do SINAN referente à "violência crônica" apontou que esse agravo ocorreu de forma repetida em grande parte dos casos, mostrando que é necessário e urgente o desenvolvimento de ações concretas e efetivas para a prevenção desses óbitos anunciados.

A violência física e a presença de diferentes tipos de violências conjugadas em um mesmo episódio, se mostraram como fatores de risco para o óbito. De fato, outro estudo em municípios do Brasil identificou que as violências mais graves estão relacionadas à sobreposição de vários tipos de violência ${ }^{3}$.

Da mesma forma, o uso de armas de fogo, de objetos perfurocortantes e a combinação de outros meios estiveram associados a maior risco de óbito. Portanto, na abordagem policial às mulheres em situação de VPI é indispensável saber se o autor tem acesso a armas de fogo e acionar os mecanismos necessários para o cumprimento da 
lei que determina a apreensão imediata de arma de fogo sob sua posse ${ }^{21}$.

Dentre as limitações do trabalho, destacaram-se aquelas relativas ao SINAN e às seleções utilizadas para o recorte deste artigo. Com relação ao SINAN, sabe-se que há subnotificação dos casos de VPI, seja pela dificuldade de identificar os casos nos atendimentos, seja pelo não registro da violência. Além disso, algumas variáveis no SINAN, como "escolaridade", "gestante" e "violência crônica" apresentaram baixa completude, podendo interferir nos resultados e ocultar situações de vulnerabilidade. Importante destacar que as bases de dados de 2015 e 2016 do SINAN, eram preliminares, assim como a de 2017 do SIM. Portanto, no SINAN poderiam ter duplicidades que não haviam sido avaliadas pelo Ministério da Saúde na consolidação da base nacional, como feito com as bases dos anos anteriores. Esse fato influenciou a escolha por trabalhar somente com as mulheres com uma notificação no período, somado ao fato de que as pesquisadoras não tiveram acesso às bases nominais para avaliação dos pares ou grupos de mulheres. Por fim, o desempenho do modelo de regressão aplicado na amostra de treino teve sensibilidade de $72 \%$ e fu- turas análises poderão encontrar amostras com melhor desempenho, o que também depende da melhoria da qualidade dos dados do SINAN.

\section{Considerações finais}

Este estudo mostrou que o relacionamento das bases de dados do SIM e do SINAN é fundamental para compreender as repercussões da VPI na vida das mulheres. Sugere-se que esse tipo de relacionamento seja feito periodicamente e que inclua outras bases de dados ou mesmo outros agravos do SINAN. Outras atividades que se fazem necessárias são a melhoria da qualidade dos dados em saúde e a formação dos profissionais para atendimento humanizado e registro adequado dos casos.

A definição de melhores modelos estatísticos para a explicação do problema e até a predição podem contribuir para a atuação da vigilância em saúde em conjunto com a rede intersetorial de enfrentamento à violência contra as mulheres. Observa-se que o setor saúde tem ciência dos casos e pode desenvolver alertas para a rede de serviços no sentido da prevenção de óbitos anunciados e evitáveis.

\section{Colaboradores}

IV Pinto trabalhou na concepção, metodologia, resultados, redação e revisão final. RTI Bernal trabalhou na metodologia, resultados e revisão final. MFM Souza trabalhou na concepção, metodologia e revisão final. DC Malta trabalhou na concepção, metodologia, resultados e revisão final. 


\section{Referências}

1. Instituto Brasileiro de Geografia e Estatística (IBGE). Objetivos de Desenvolvimento Sustentável [Internet]. [acessado 20 Jan 2020]. Disponível em: https://ods. ibge.gov.br/

2. Stöckl H, Devries K, Rotstein A, Abrahams N, Campbell J, Watts C, Moreno CG. The global prevalence of intimate partner homicide: a systematic review. Lancet 2013; 382(9895):859-865.

3. Schraiber LB, D’Oliveira AFPL, França-Junior I, Diniz S, Portella AP, Ludermir AB, Valença O, Couto MT. Prevalência da violência contra a mulher por parceiro íntimo em regiões do Brasil. Rev Saúde Pública 2007; 41(5):797-807.

4. Minayo MCS, Souza ER, Silva MMA, Assis SG. Institucionalização do tema da violência no SUS: avanços e desafios. Cien Saude Colet 2018; 23(6):2007-2016.

5. Silva MMA, Mascarenhas MDM, Lima CM, Malta DC, Monteiro RA, Freitas MG, Melo ACM, Bahia CA, Bernal RTI. Perfil do inquérito de violências e acidentes em serviços sentinela de urgência e emergência. Epidemiol Serv Saude 2017; 26(1):183-194.

6. Brasil. Ministério da Saúde (MS). Secretaria de Vigilância em Saúde. Departamento de Vigilância de Doenças e Agravos não Transmissíveis e Promoção da Saúde. Saúde Brasil 2018 uma análise de situação de saúde e das doenças e agravos crônicos: desafios e perspectivas. Brasília: MS; 2019.

7. Brasil. Ministério da Saúde (MS). Departamento de Informática do SUS. CID-10 [Internet]. [acessado 2019 Set 3]. Disponível em: http://datasus1.saude.gov. $\mathrm{br} /$ sistemas-e-aplicativos/cadastros-nacionais/cid-10

8. Brasil. Ministério da Saúde (MS). Departamento de Informática do SUS [Internet]. [acessado 2020 Jun 1]. Disponível em http://tabnet.datasus.gov.br/cgi/deftohtm.exe?sim/cnv/obt10uf.def

9. Santos HG. Comparação da performance de algoritmos de machine learning para análise preditiva em saúde pública e medicina [tese]. São Paulo: Universidade de São Paulo; 2018.

10. R Core Team. R: A language and environment for statistical computing. Vienna: R Foundation for Statistical Computing; 2020.

11. Del Priore M. Ao sul do corpo: condição feminina, maternidade e mentalidades no Brasil Colônia. São Paulo: Editora UNESP; 2009.

12. Campbell J, Webster D, Koziol-McLain J, Block C, Campbell D, Curry MA, Gary F, Glass N, McFarlane J, Sachs C, Sharps P, Ulrich Y, Wilt AS, Manganello J, Xu $\mathrm{X}$, Schollenberger J, Frye V, Laughon K. Risk factors for femicide in abusive relationships: results from a multisite case control study. Am J Public Health 2003; 93(7):1089-1097.
13. ONU Mulheres. Diretrizes Nacionais Feminicídio. Investigar, Processar e Julgar com Perspectiva de Gênero as Mortes Violentas de Mulheres. Brasília: ONU Mulheres; 2016.

14. Gollub EL, Gardner M. Firearm legislation and firearm use in female intimate partner homicide using National Violent Death Reporting System data. Preventive Med 2019; 118:216-219.

15. Rafael RMR, Moura ATMS. Violência física grave entre parceiros íntimos como fator de risco para inadequação no rastreio do câncer de colo de útero. Cad Saúde Pública 2017; 33(12):e00074216.

16. Johnson MP. A typology of domestic violence. United States of America: Northeastern University Press; 2008.

17. Brancaglioni BCA, Fonseca RMGS. Intimate partner violence in adolescence: an analysis of gender and generation. Rev Bras Enferm 2016; 69(5):890-898.

18. Mascarenhas MDM, Tomaz GR, Meneses GMS, Rodrigues MTP, Preira VOM, Corassa FB. Análise das notificações de violência por parceiro íntimo contra mulheres, Brasil, 2011-2017. Rev Bras Epidemiol 2020, 23(Supl. 1):E200007.

19. Moraes CL, Arana FDN, Reichenheim ME. Violência física entre parceiros íntimos na gestação como fator de risco para a má qualidade do pré-natal Rev Saúde Pública 2010; 44(4):667-676.

20. Brasil. Ministério da Saúde (MS). Secretaria de Vigilância em Saúde. Departamento de Vigilância de Doenças e Agravos não Transmissíveis e Promoção da Saúde. Saúde Brasil 2017: uma análise da situação de saúde e os desafios para o alcance dos objetivos de desenvolvimento sustentável. Brasília: MS; 2018.

21. Brasil. Presidência da República. Secretaria Geral. Subchefia para Assuntos Jurídicos. Lei no 13.880, de 08 de outubro de 2019. Altera a Lei no 11.340 , de 7 de agosto de 2006 (Lei Maria da Penha), para prever a apreensão de arma de fogo sob posse de agressor em casos de violência doméstica, na forma em que especifica. Diário Oficial da União; 2019.

Artigo apresentado em 04/01/2021

Aprovado em 05/01/2021

Versão final apresentada em 07/01/2021

Editores-chefes: Maria Cecília de Souza Minayo, Romeu Gomes, Antônio Augusto Moura da Silva 
Errata

p. 975

Onde se lê

ARTIGO ARTICLE

Leia-se

TEMAS LIVRES FREE THEMES

Revista Ciência \& Saúde Coletiva 2021; 26(5): 1991-1991. 